Research Article

\title{
Bending Analysis of Circular Thin Plates Resting on Elastic Foundations Using Two Modified Vlasov Models
}

\author{
Feng Yue $\mathbb{D}^{D}$, Fusheng Wang $\mathbb{D}^{D}$, Senqing Jia, Ziyan Wu, and Zhen Wang \\ School of Mechanics, Civil Engineering and Architecture, Northwestern Polytechnical University, Xi'an 710072, China \\ Correspondence should be addressed to Fusheng Wang; fswang@nwpu.edu.cn
}

Received 2 April 2020; Revised 28 May 2020; Accepted 12 June 2020; Published 6 July 2020

Academic Editor: Nerio Tullini

Copyright (c) 2020 Feng Yue et al. This is an open access article distributed under the Creative Commons Attribution License, which permits unrestricted use, distribution, and reproduction in any medium, provided the original work is properly cited.

The influence of soil heterogeneity is studied on the bending of circular thin plates using two modified Vlasov foundation models. The model parameters are determined reasonably using an iterative technique. According to the principle of minimum potential energy and considering transversely isotropic soils and Gibson soils, the governing differential equations and boundary conditions for circular thin plates on two modified Vlasov foundations are derived using a variational approach, respectively. The determination of attenuation parameters is a difficult problem, which has hindered the further application of the Vlasov foundation model. The equation that must be satisfied by the attenuation parameter is determined, and an iterative method is used to solve the problem. A comparative analysis is conducted between two modified Vlasov models and the traditional Vlasov model. The results show that the governing equations and boundary conditions for circular thin plates resting on two modified foundations are consistent with those for a circular thin plate on traditional two-parameter foundation after degradation. The accuracy and reliability of the proposed solutions are demonstrated by comparing the obtained results with those reported in the literature. The heterogeneity of soils, including the transversely isotropic soils and Gibson soils, has a certain effect on characteristic parameters of the foundation models as well as the deformations and internal forces of circular thin plates. The present study could be employed as a reference for future engineering designs.

\section{Introduction}

Plates supported directly by the soil continuum are widely used in structural engineering. They are the basic components of highways, bridges, high-rise building foundations, and other structures. The behaviour of the plate when it carries external loads is influenced by the foundation, and the behaviour of the foundation is in turn influenced by the action of the plate under load. In order to study the practical problems of beams, plates, and shells on elastic foundations in engineering, scholars have put forward many mathematical models. It is very important to calculate and analyse these models accurately. The existing elastic foundation models include the Winkler foundation model [1], two-parameter elastic model [2], and elastic continuous medium foundation model [3], each of which has its own characteristics in accordance with the corresponding hypotheses. The two-parameter foundation model has the advantages of a simple mathematical process and a perfect theory. As an example of the two-parameter foundation model, the Vlasov foundation model has all of the advantages of a continuum approach as well as the simplicity of the coupled spring model. Compared to standard numerical models such as FEM (Finite Element Modelling), analytical solutions have more advantages in terms of understanding the essential aspects of physics and mechanics.

Many researchers have worked on this problem, which is described as "beams, plates, and shells on elastic foundations." First of all, in terms of the Vlasov foundation model, Vallabhan et al. [4-6] analysed the governing equations and boundary conditions for the bending of rectangular plates and beams resting on a refined Vlasov foundation, using variational principles. Höller et al. [7] rigorously derived a 
theory for elastically supported thin plates with arbitrary boundary conditions, based on the Principle of Virtual Power. Ozgan [8] carried out the dynamic analysis of thick plates including deep beams on elastic foundations using a modified Vlasov foundation model. Wang et al. [9] developed a thermoelastic analysis of pavement plates on an elastic Winkler foundation, based on the Kirchhoff hypothesis and a Vlasov-type of structural analysis. Secondly, with regard to the transversely isotropic foundation model, Ai and Feng [10] analysed laterally loaded fixed-head pile groups using BEM (Boundary Element Method), based on an analytical layer-element solution of multilayered transversely isotropic soils. With the inverse Hankel integration transform, the solution of stress and displacement for transversely isotropic foundation under several common loads was obtained [11]. Liang and Shatnawi [12] presented a series of charts, based on extensive FE (Finite Element) parametric studies along with nonlinear regression analysis of FE simulation results, for estimating the subgrade reaction modulus using five elastic constants of a transversely isotropic rock mass. Finally, to analyse structures resting on elastic foundations based on Gibson soils, some useful conclusions concerning displacements and stresses in a nonhomogeneous elastic half-space were drawn [13]. Eisenberger and Clastornik $[14,15]$ studied vibrations and buckling of a beam resting on a variable Winkler elastic foundation.

Because of the difficulties in mathematics and mechanics, there are some problems in the previous research on the classical subject of foundation structure. For example, the classical two-parameter foundation model considers that the elastic layer is homogeneous and isotropic. In fact, soil is a kind of complex multiphase discrete medium. On one hand, tests show that many foundations are close to the transversely isotropic soils, which can be regarded as isotropic in the horizontal direction. However, the properties of foundation soil along the vertical direction are quite different. The research progress in transversely isotropic elastic foundations has been slow owing to the large number of elastic constants and the complex derivation process. On the other hand, the elasticity modulus is considered to vary linearly along the depth of foundations. Thus, Gibson foundation is more in line with the actual situation. As another example, even though the traditional two-parameter model for plates resting on elastic foundations represents the interaction between the plates and the foundation better than the Winkler model, it requires the estimation of a third parameter $\gamma$, which represents the distribution of displacements within the foundation. Yang [16] thought that there was no way to provide a method to calculate the undetermined parameter value. Vallabhan and Das [17] deduced that the decay parameter was a function of some nondimensional parameters of the beams, the elastic foundations, and the mode of loads, using variational principles. Jones and Xenophontos [18] established a relationship between the attenuation parameter and the displacement characteristics but did not actually determine its value.
The soil inhomogeneity is not considered in the existing Vlasov foundation model. Moreover, only empirical or experimental values have been used for model parameters such as the attenuation parameter. Therefore, in the present study, circular thin plates resting on two modified Vlasov foundations are analysed based on heterogeneous soils with transverse isotropy and Gibson characteristics, respectively. The static bending of a circular thin plate is analysed systematically on the interaction between foundation soils and circular thin plates. In addition, the influence of heterogeneous soils on the bending of circular thin plates resting on two modified foundations is examined. Firstly, the governing equations and boundary conditions for a circular thin plate resting on two modified Vlasov foundations are established, according to the principle of energy variation. At the same time, the equation that must be satisfied by the attenuation parameter is determined. Secondly, the similarities and differences between the traditional Vlasov model and two modified Vlasov models are analysed. Then, the two correct parameter values are calculated using attenuation parameter values obtained through an iterative method. Finally, the relatively accurate deflections and internal forces of circular thin plate are obtained.

\section{Foundation Model}

2.1. Transversely Isotropic Foundation. The foundation is approximately regarded as isotropic in the horizontal direction; however, the difference between the vertical and the horizontal directions is large. The hypothesis of homogeneity and isotropy cannot reflect the reality of the foundations. Nevertheless, the transversely isotropic elastic model can reflect the continuity of stresses and deformations of foundations spreading around the base. Therefore, a transversely isotropic elastic model is more suitable for the actual situation of the foundation. Considering these characteristics of foundations, it can better study the stability of foundations and the mechanism of stress transmission.

As shown in Figure 1, the radius of the circular thin plate is denoted as $a$ and the thickness is $h$. The radius of a uniform surface load $q$ is represented by $b$ and the foundation depth is $H$. The constitutive relation of the transversely isotropic elastic foundation is

$$
\begin{cases}\sigma_{r}=C_{11} \varepsilon_{r}+C_{12} \varepsilon_{\theta}+C_{13} \varepsilon_{z}, & \tau_{r \theta}=C_{66} \gamma_{r \theta}, \\ \sigma_{\theta}=C_{12} \varepsilon_{r}+C_{11} \varepsilon_{\theta}+C_{13} \varepsilon_{z}, & \tau_{\theta z}=C_{44} \gamma_{\theta z}, \\ \sigma_{z}=C_{13} \varepsilon_{r}+C_{13} \varepsilon_{\theta}+C_{33} \varepsilon_{z}, & \tau_{z r}=C_{44} \gamma_{z r},\end{cases}
$$

where $\sigma_{r}, \sigma_{\theta}, \sigma_{z}, \tau_{r \theta}, \tau_{\theta z}$, and $\tau_{z r}$ are the stresses in the foundation, correspondingly; $\varepsilon_{r}, \varepsilon_{\theta}, \varepsilon_{z}, \gamma_{r \theta}, \gamma_{\theta z}, \gamma_{z r}$ are the strains in the foundation; and $C_{i j}$ are physical property constants of the transversely isotropic foundation. As long as the five independent engineering elastic constants $\left(E_{T 1}, \mu_{T 1}, E_{T 2}, \mu_{T 2}\right.$, and $\left.G_{T 2}\right)$ of the transversely isotropic body are determined, the values of $C_{i j}$ can be determined. The values of $C_{i j}$ have the following relationship with the material elastic constants commonly used in engineering: 


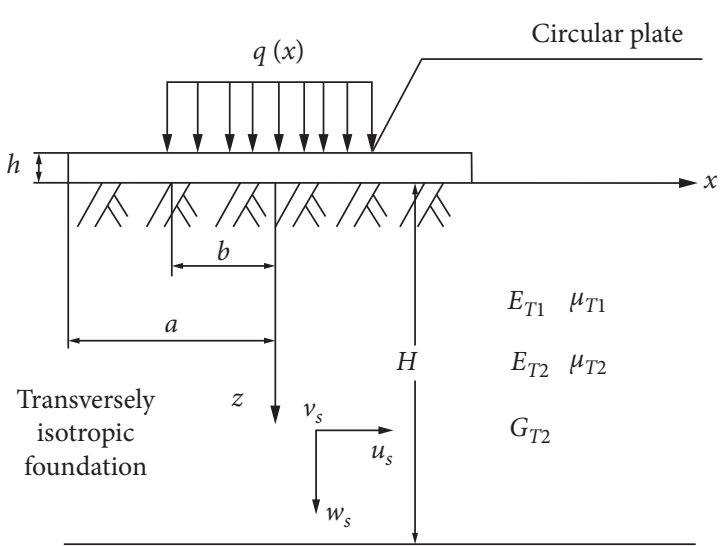

Rigid base

Figure 1: Plate on a transversely isotropic foundation.

$$
\begin{aligned}
& \left\{\begin{array}{l}
C_{11}=\lambda_{1} N\left(1-N \mu_{T 2}^{2}\right), \quad C_{12}=\lambda_{1} N\left(\mu_{T 1}+N \mu_{T 2}^{2}\right), \\
C_{13}=\lambda_{1} N \mu_{T 2}\left(1+\mu_{T 1}\right), \quad C_{33}=\lambda_{1}\left(1-\mu_{T 1}^{2}\right),
\end{array}\right. \\
& \left\{\begin{array}{l}
C_{44}=G_{T 2} \\
C_{66}=\frac{E_{T 1}}{2\left(1+\mu_{T 1}\right)} \\
N=\frac{E_{T 1}}{E_{T 2}}, \\
\lambda_{1}=\frac{E_{T 2}}{\left(1+\mu_{T 1}\right)\left(1-\mu_{T 1}-2 N \mu_{T 2}^{2}\right)}
\end{array}\right.
\end{aligned}
$$

where $E_{T 1}$ and $\mu_{T 1}$ are the elasticity modulus and Poisson's ratio in the isotropic plane, $E_{T 2}, \mu_{T 2}$ denote the elasticity modulus and Poisson's ratio in the normal direction of the isotropic plane, and $G_{T 2}$ represents the shear modulus in the plane perpendicular to the isotropic plane. Moreover, $\lambda_{1}$ and $N$ are common parameters of the transversely isotropic elastic foundation in engineering.

2.2. Gibson Foundation. In engineering, owing to the sedimentation of soils, the elasticity modulus of heterogeneous soils changes with the depth. Considering the heterogeneity of foundation soils, it is assumed that the soil is a Gibson foundation whose elasticity modulus changes linearly in accordance with the depth. Let the elasticity modulus at the top and bottom of the foundation be $E_{G 1}$ and $E_{G 2}$, respectively. The dimensionless parameter $\lambda_{2}$ is introduced.

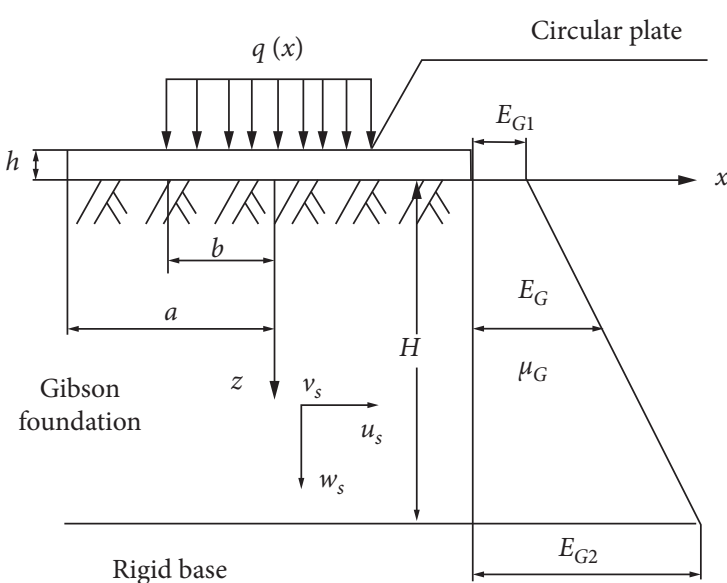

Figure 2: Plate on a Gibson elastic foundation.

$$
\lambda_{2}=\frac{E_{G 1}}{E_{G 2}}
$$

As shown in Figure 2, $\mu_{G}$ is Poisson's ratio of the Gibson foundation, and the elasticity modulus $E_{G}$ at depth $z$ is

$$
E_{G}=E_{G 2}\left[\lambda_{2}+\left(1-\lambda_{2}\right) \frac{z}{H}\right] \text {. }
$$

\section{Theoretical Derivation}

3.1. Potential Energy of System. As shown in Figures 1 and 2, a circular thin plate resting on heterogeneous soils is investigated using two modified Vlasov foundation models.

The total potential energy of the plate-foundation system is as follows:

$$
U_{i}=U_{p}+U_{s i}+U_{q}, \quad i=1,2,
$$

where $U_{i}, U_{p}, U_{s i}$, and $U_{q}$ are the total potential energy function, the deformation potential energy of a circular thin plate, the deformation potential energy of two kinds of modified foundations, and the potential energy of the external force, respectively. When $i$ is equal to 1 , the physical quantities $U_{1}$ and $U_{s 1}$ represent the potential energy of the transversely isotropic plate-foundation system and the deformation potential energy of the transversely isotropic foundation, respectively. When $i$ is equal to 2, the physical quantities $U_{2}$ and $U_{s 2}$ represent the potential energy of the Gibson plate-foundation system and the deformation potential energy of the Gibson foundation, respectively. In this paper, unless otherwise specified, the symbols with subscript 1 are the variables for the transversely isotropic foundation, and the symbols with subscript 2 are the variables for the Gibson elastic foundation.

The details are as follows: 


$$
U_{p}=\frac{D}{2} \iint_{\Omega}\left\{\left(\frac{\partial^{2} w}{\partial r^{2}}+\frac{1}{r} \frac{\partial w}{\partial r}+\frac{1}{r^{2}} \frac{\partial^{2} w}{\partial \theta^{2}}\right)^{2}-2(1-\mu)\left[\frac{\partial^{2} w}{\partial r^{2}}\left(\frac{1}{r} \frac{\partial w}{\partial r}+\frac{1}{r^{2}} \frac{\partial^{2} w}{\partial \theta^{2}}\right)-\left(\frac{1}{r} \frac{\partial^{2} w}{\partial r \partial \theta}-\frac{1}{r^{2}} \frac{\partial w}{\partial \theta}\right)^{2}\right]\right\} r \mathrm{~d} r \mathrm{~d} \theta
$$

where $D$ is the bending stiffness of the plate, $E$ and $\mu$ represent the modulus of elasticity and Poisson's ratio respectively, $w$ denotes the deflection of the circular thin plate, and $\Omega$ represents the region of the circular thin plate.

$$
\begin{aligned}
D & =\frac{E h^{3}}{12\left(1-\mu^{2}\right)}, \\
U_{s i} & =\frac{1}{2} \int_{0}^{H} \int_{0}^{2 \pi} \int_{0}^{+\infty}\left(\sigma_{r} \varepsilon_{r}+\sigma_{\theta} \varepsilon_{\theta}+\sigma_{z} \varepsilon_{z}+\tau_{r \theta} \gamma_{r \theta}+\tau_{\theta z} \gamma_{\theta z}+\tau_{z r} \gamma_{z r}\right) r \mathrm{~d} r \mathrm{~d} \theta \mathrm{d} z, \quad i=1,2 .
\end{aligned}
$$

Here, the terms $\left(u_{s}, v_{s}, w_{s i}\right)$ represent the displacements in the $x, y$, and $z$ directions, respectively, in the foundation. $w_{s 1}$ and $w_{s 2}$ represent the deflection of the transversely isotropic elastic foundation and that of the Gibson elastic foundation, respectively. From practical considerations, it can be shown that the horizontal displacements $u_{s}$ and $v_{s}$ in the foundation are negligible compared with the vertical displacements $w_{s i}$ in the $z$ direction.

$$
\begin{aligned}
u_{s}(r, \theta, z) & =0, \\
v_{s}(r, \theta, z) & =0, \\
w_{s i}(r, \theta, z) & =w_{s i}(r, \theta) \varphi_{i}(z), \quad i=1,2,
\end{aligned}
$$

where $w_{s i}(r, \theta)$ is the displacement at the foundation surface and $\varphi_{i}(z)$ is the attenuation function. On the contact surface between the circular thin plate and foundations, the displacement is continuous. That is, the values of $\varphi_{i}(0)$ and $\varphi_{i}(H)$ are set equal to 1 and 0 such that $w_{s i}(r, \theta)$ becomes the deflection of the soil at the surface.

The constitutive relations of the two modified foundation models are different; however, the geometric relations are the same. The expressions for two kinds of foundation deformation potential energy $U_{s i}(i=1,2)$ are obtained

$$
\begin{aligned}
& U_{s 1}=\frac{1}{2} \int_{0}^{H} \int_{0}^{+\infty} \int_{0}^{2 \pi}\left\{C_{33} w_{s 1}^{2}\left(\frac{\mathrm{d} \varphi_{1}}{d z}\right)^{2}+C_{44}\left[\left(\frac{\partial w_{s 1}}{\partial r}\right)^{2}+\frac{1}{r^{2}}\left(\frac{\partial w_{s 1}}{\partial \theta}\right)^{2}\right] \varphi_{1}^{2}\right\} r \mathrm{~d} r \mathrm{~d} \theta \mathrm{d} z \\
& =\frac{1}{2} \int_{0}^{+\infty} \int_{0}^{2 \pi}\left\{k_{1} w_{s 1}^{2}+G_{p 1}\left[\left(\frac{\partial w_{s 1}}{\partial r}\right)^{2}+\frac{1}{r^{2}}\left(\frac{\partial w_{s 1}}{\partial \theta}\right)^{2}\right]\right\} r \mathrm{~d} r \mathrm{~d} \theta \\
& U_{s 2}=\frac{1}{2} \int_{0}^{H} \int_{0}^{+\infty} \int_{0}^{2 \pi}\left\{\frac{E_{G}\left(1-\mu_{G}\right)}{\left(1+\mu_{G}\right)\left(1-2 \mu_{G}\right)} w_{s 2}^{2}\left(\frac{\mathrm{d} \varphi_{2}}{\mathrm{~d} z}\right)^{2}+\frac{E_{G}}{2\left(1+\mu_{G}\right)}\left[\left(\frac{\partial w_{s 2}}{\partial r}\right)^{2}+\frac{1}{r^{2}}\left(\frac{\partial w_{s 2}}{\partial \theta}\right)^{2}\right] .\right. \\
& \left.\varphi_{2}^{2}\right\} r \mathrm{~d} r \mathrm{~d} \theta \mathrm{d} z=\frac{1}{2} \int_{0}^{+\infty} \int_{0}^{2 \pi}\left\{k_{2} w_{s 2}^{2}+G_{p 2}\left[\left(\frac{\partial w_{s 2}}{\partial r}\right)^{2}+\frac{1}{r^{2}}\left(\frac{\partial w_{s 2}}{\partial \theta}\right)^{2}\right]\right\} r \mathrm{~d} r \mathrm{~d} \theta \\
& \left\{\begin{array}{l}
k_{1}=\int_{0}^{H} C_{33}\left(\frac{\mathrm{d} \varphi_{1}}{\mathrm{~d} z}\right)^{2} \mathrm{~d} z \\
G_{p 1}=\int_{0}^{H} C_{44} \varphi_{1}^{2} \mathrm{~d} z
\end{array}\right. \\
& \left\{\begin{array}{l}
k_{2}=\int_{0}^{H} \frac{E_{G 2}\left(1-\mu_{G}\right)}{\left(1+\mu_{G}\right)\left(1-2 \mu_{G}\right)}\left[\lambda_{2}+\left(1-\lambda_{2}\right) \frac{z}{H}\right]\left(\frac{\mathrm{d} \varphi_{2}}{\mathrm{~d} z}\right)^{2} \mathrm{~d} z, \\
G_{p 2}=\int_{0}^{H} \frac{E_{G 2}}{2\left(1+\mu_{G}\right)}\left[\lambda_{2}+\left(1-\lambda_{2}\right) \frac{z}{H}\right] \varphi_{2}^{2} \mathrm{~d} z,
\end{array}\right.
\end{aligned}
$$


where $k_{1}$ and $G_{p 1}$ are the modulus of the subgrade reaction and shear coefficient of the transversely isotropic two-parameter foundation, respectively. Additionally, $k_{2}$ and $G_{p 2}$ denote the modulus of the subgrade reaction and shear coefficient of the Gibson two-parameter foundation, respectively.

Moreover, external force potential energy is

$$
U_{q}=-\iint_{\Omega_{o}} q w_{s i} r \mathrm{~d} r \mathrm{~d} \theta, \quad i=1,2,
$$

where $\Omega_{o}$ represents the area under a uniformly distributed load.

3.2. The Governing Equations. The governing differential equations for the circular thin plate and those for the foundation surface outside the plate domain can be derived using principles of variational calculus:

$$
\begin{aligned}
D \nabla^{4} w-G_{p 1} \nabla^{2} w+k_{1} w & =q, \\
-G_{p 1} \nabla^{2} w_{s 1}+k_{1} w_{s 1} & =0, \\
D \nabla^{4} w-G_{p 2} \nabla^{2} w+k_{2} w & =q, \\
-G_{p 2} \nabla^{2} w_{s 2}+k_{2} w_{s 2} & =0 .
\end{aligned}
$$

Similarly, the equations for the attenuation function $\varphi_{i}$ are obtained as follows:

$$
\begin{aligned}
-m_{i} \frac{\mathrm{d}^{2} \varphi_{i}}{\mathrm{~d} z^{2}}+n_{i} \varphi_{i} & =0, \quad i=1,2, \\
\varphi_{i}(0) & =1, \\
\varphi_{i}(H) & =0,
\end{aligned}
$$

where

$$
\begin{aligned}
& \left\{\begin{array}{l}
m_{1}=\int_{0}^{+\infty} \int_{0}^{2 \pi} C_{33} w_{s 1}^{2} r \mathrm{~d} r \mathrm{~d} \theta, \\
n_{1}=\int_{0}^{+\infty} \int_{0}^{2 \pi} C_{44}\left[\left(\frac{\partial w_{s 1}}{\partial r}\right)^{2}+\frac{1}{r^{2}}\left(\frac{\partial w_{s 1}}{\partial \theta}\right)^{2}\right] r \mathrm{~d} r \mathrm{~d} \theta,
\end{array}\right. \\
& \left\{\begin{array}{l}
m_{2}=\int_{0}^{+\infty} \int_{0}^{2 \pi} \frac{E_{G 2}\left(1-\mu_{G}\right)}{\left(1+\mu_{G}\right)\left(1-2 \mu_{G}\right)} w_{s 2}^{2} r \mathrm{~d} r \mathrm{~d} \theta, \\
n_{2}=\int_{0}^{+\infty} \int_{0}^{2 \pi} \frac{E_{G 2}}{2\left(1+\mu_{G}\right)}\left[\left(\frac{\partial w_{s 2}}{\partial r}\right)^{2}+\frac{1}{r^{2}}\left(\frac{\partial w_{s 2}}{\partial \theta}\right)^{2}\right] r \mathrm{~d} r \mathrm{~d} \theta,
\end{array}\right. \\
& \alpha_{i}^{2}=\frac{k_{i}}{G_{p i}}, \quad i=1,2,
\end{aligned}
$$

where $\alpha_{i}(i=1,2)$ represents the process parameters of the two refined Vlasov elastic foundation models. In the subsequent solving process, let $\alpha$ represent $\alpha_{1}$ and $\alpha_{2}$; that is, there is no need to distinguish them.

According to the governing differential equation (12) and boundary conditions (13), the attenuation function $\varphi_{i}(z)$ and attenuation parameter $\gamma_{i}$ are derived:

$$
\begin{array}{rr}
\varphi_{i}(z)=\frac{\sinh \left[\gamma_{i}(1-z / H)\right]}{\sinh \gamma_{i}}, & i=1,2, \\
\left(\frac{\gamma_{i}}{H}\right)^{2}=\frac{n_{i}}{m_{i}}, & i=1,2 .
\end{array}
$$

The governing equations and boundary conditions for a circular thin plate resting on two modified foundations are consistent with those for the plate on the traditional two-parameter foundation after degradation. Mathematically, the two modified elastic foundation models are also equivalent and differ only in terms of the definition of the foundation parameters.

3.3. Model Parameters. The Winkler model suffers the setback of noninteraction between the lateral spring thereby resulting into an unreliable result. So the two-parameter elastic foundation is developed to account for the interaction. While it is easy to establish quite accurately the stiffness characteristics of beams or plates, the parameters governing the behaviour of the subsoil or elastic foundations are indeed hard to model. In many calculation examples of the interaction between the foundations and structures, the values of the model parameters depend on experience or tests.

In this paper, a mathematical model is developed for the analysis of circular thin plates on two modified Vlasov elastic foundations using three parameters, such as $k_{i}, G_{p i}$, and $\gamma_{i}$, which are related to the material properties and geometry of the foundation continuum for a given thin plate and the loading on it. Figure 3 shows an iterative flow chart of attenuation parameter $\gamma_{1}$ in the transversely isotropic elastic foundation. Figure 4 presents an iterative flow chart of attenuation parameter $\gamma_{2}$ in the Gibson elastic foundation. Both the parameter $k_{i}$, which represents the modulus of subgrade reaction, and the parameter $G_{p i}$, which represents the shear deformation of the soil, are uniquely calculated using the modulus of elasticity, Poisson's ratio, and the geometry and deformation of the elastic foundation. The arbitrary aspect of the Vlasov elastic foundation model has been mathematically removed through the development of a computational technique for determining the value of a third parameter $\gamma_{i}$, which reflects the variation of the soil deformation.

\section{Solution Method}

4.1. Solution. In the case of axisymmetric, the deformation of the circular thin plate resting on the elastic foundation is axisymmetric. Thus, $w$ is only the function of the radius $r$, independent of the angle $\theta$. The governing equation for a circular thin plate on two modified Vlasov elastic foundations is as follows:

$$
D \nabla^{4} w(r)-G_{p i} \nabla^{2} w(r)+k_{i} w(r)=q(r), \quad i=1,2 .
$$

In theory, three groups of the governing equations need to be solved. However, by comparing these Vlasov elastic foundation models, it is found that no difference exists in the form of governing equations and boundary conditions; however, the specific expressions for the characteristic parameters are changed. Therefore, the specific process of 


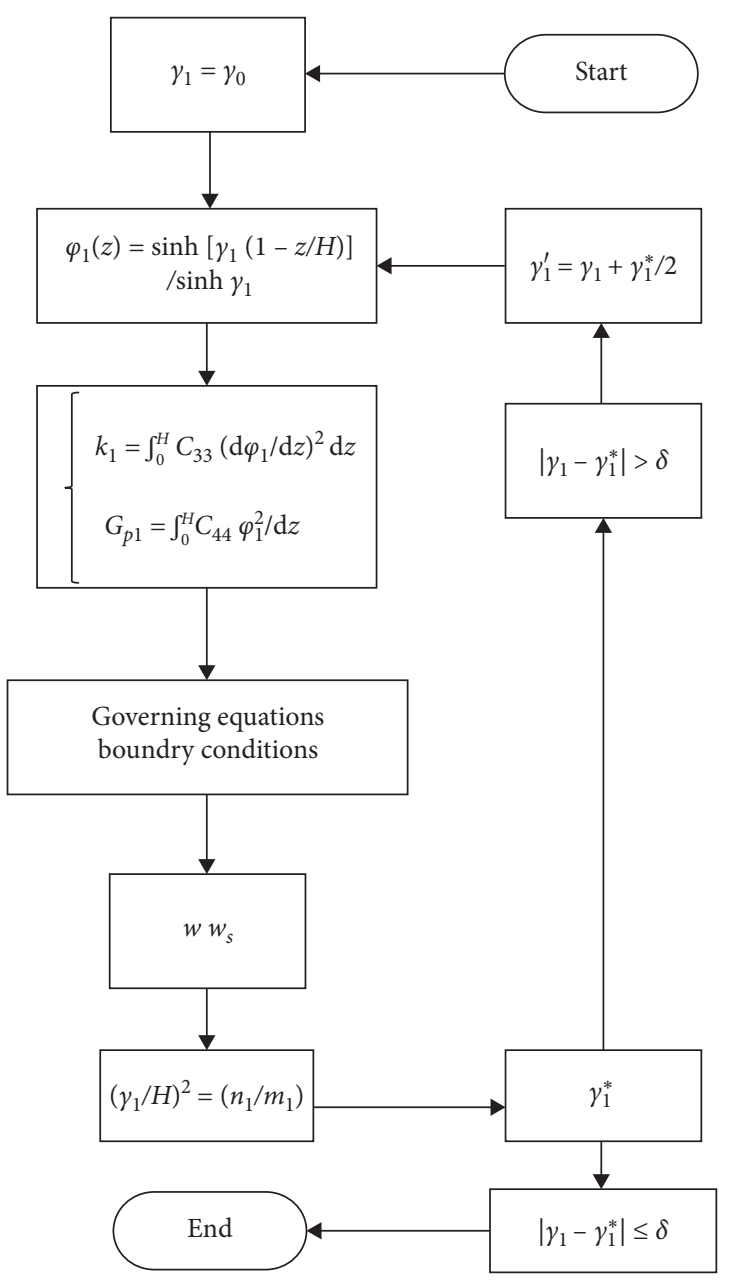

FIGURE 3: Iterative flow chart of the attenuation parameter for the transversely isotropic foundation.

solving the bending problem of circular thin plates on these Vlasov elastic foundation models is similar.

To simplify the mathematical operation, let $k$ represent $k_{1}, k_{2}$ and $G_{p}$ represent $G_{p 1}, G_{p 2}$; that is, there is no formal distinction between $k_{1}\left(G_{p 1}\right)$ and $k_{2}\left(G_{p 2}\right)$. Generally, the solution of the differential equation (16) can be written as the sum of the homogeneous solution and the specific solution. The specific solution depends on the shape of the load. The homogeneous solution is that of the following homogeneous equation, namely,

$$
\nabla^{4} w(r)-\frac{G_{p}}{D} \nabla^{2} w(r)+\frac{k}{D} w(r)=0 .
$$

The above formula can be transformed into

$$
\left(\nabla^{2}-\eta_{1}\right)\left(\nabla^{2}-\eta_{2}\right) w(r)=0
$$

where

$$
\left\{\begin{array}{l}
\eta_{1} \\
\eta_{2}
\end{array}=\frac{1}{2}\left\{\frac{G_{p}}{D} \pm\left[\left(\frac{G_{p}}{D}\right)^{2}-\frac{4 k}{D}\right]^{1 / 2}\right\} .\right.
$$

The solution of equation (17) can be written in various forms using the modified Bessel function of real independent variables and the Bessel function of virtual independent variables. In this study, the solution proposed by Vlasov and Leontiev for calculations is employed as follows [19]:

$$
\begin{gathered}
w(r)=B_{1} J_{0}\left(\xi_{1}^{1 / 2} \frac{r}{l}\right)+B_{2} H_{0}^{(1)}\left(\xi_{1}^{1 / 2} \frac{r}{l}\right)+B_{3} J_{0}\left(\xi_{2}^{1 / 2} \frac{r}{l}\right)+B_{4} H_{0}^{(2)}\left(\xi_{2}^{1 / 2} \frac{r}{l}\right) \\
\left\{\begin{array}{l}
\xi_{1} \\
\xi_{2}
\end{array}=\frac{l^{2}}{2}\left\{-\frac{G_{p}}{D} \pm\left[\left(\frac{G_{p}}{D}\right)^{2}-\frac{4 k}{D}\right]^{1 / 2}\right\}\right. \\
l^{4}=\frac{D}{k}
\end{gathered}
$$

where $B_{1}, B_{2}, B_{3}$, and $B_{4}$ are arbitrary constants, $J_{0}$ denotes the Bessel function of order zero, and $H_{0}^{(n)}$ represents the Hankel function of order $n$.

Since both the Bessel function and Hankel function in equation (20) are complex, the constants $B_{1}, B_{2}, B_{3}$, and $B_{4}$ must be complex to make the deflections of a circular thin plate real. Then equation (20) can be expressed as a real function:

$$
\begin{aligned}
& w(r)=d_{1} s_{0}\left(\frac{r}{l}\right)+d_{2} t_{0}\left(\frac{r}{l}\right)+d_{3} f_{0}\left(\frac{r}{l}\right)+d_{4} g_{0}\left(\frac{r}{l}\right), \\
& s_{0}\left(\frac{r}{l}\right)=\operatorname{Re} J_{0}\left(\xi_{1}^{1 / 2} \frac{r}{l}\right)=\frac{1}{2}\left[J_{0}\left(\xi_{1}^{1 / 2} \frac{r}{l}\right)+J_{0}\left(\xi_{1}^{1 / 2} \frac{r}{l}\right)\right], \\
& t_{0}\left(\frac{r}{l}\right)=\operatorname{Im} J_{0}\left(\xi_{2}^{1 / 2} \frac{r}{l}\right)=\frac{1}{2 i}\left[J_{0}\left(\xi_{1}^{1 / 2} \frac{r}{l}\right)-J_{0}\left(\xi_{2}^{1 / 2} \frac{r}{l}\right)\right], \\
& \left\{\begin{array}{l}
f_{0}\left(\frac{r}{l}\right)=\operatorname{Re} H_{0}^{(1)}\left(\xi_{1}^{1 / 2} \frac{r}{l}\right)=\frac{1}{2}\left[H_{0}^{(1)}\left(\xi_{1}^{1 / 2} \frac{r}{l}\right)+H_{0}^{(2)}\left(\xi_{2}^{1 / 2} \frac{2}{l}\right)\right], \\
g_{0}\left(\frac{r}{l}\right)=\operatorname{Im} H_{0}^{(2)}\left(\xi_{2}^{1 / 2} \frac{r}{l}\right)=\frac{1}{2 i}\left[H_{0}^{(1)}\left(\xi_{1}^{1 / 2} \frac{r}{l}\right)-H_{0}^{(2)}\left(\xi_{2}^{1 / 2} \frac{r}{l}\right)\right],
\end{array}\right.
\end{aligned}
$$

where $s_{0}(r / l)$ and $f_{0}(r / l)$ represent the real parts of the zeroorder Bessel and Hankel functions and $t_{0}(r / l)$ and $g_{0}(r / l)$ represent the imaginary parts, respectively. Because these functions are all real, the undetermined constants $d_{1}, d_{2}, d_{3}$, and $d_{4}$ are also real.

The loaded area $(0 \leq r \leq b)$ and unloaded area $(b \leq r \leq a)$ of the circular thin plate and the unloaded area $(r \geq a)$ of the soil medium are considered, respectively. Additionally, $w_{1}^{*}(r)$ and $w_{2}^{*}(r)$ represent the deflections of the circular thin plate in the loaded area and the unloaded areas, respectively, and $w_{3}^{*}(r)$ denotes the surface deflection of the soil medium outside the circular thin plate:

$$
\begin{aligned}
D \nabla^{4} w_{1}^{*}(r)-G_{p} \nabla^{2} w_{1}^{*}(r)+k w_{1}^{*}(r)=q, & 0 \leq r \leq b, \\
D \nabla^{4} w_{2}^{*}(r)-G_{p} \nabla^{2} w_{2}^{*}(r)+k w_{2}^{*}(r)=0, & b \leq r<a, \\
-G_{p} \nabla^{2} w_{3}^{*}(r)+k w_{3}^{*}(r)=0, & a<r<+\infty .
\end{aligned}
$$

The special solution for $w_{1}^{*}(r)$ corresponding to a uniform load $q$ is $q / k$, while the special solutions for $w_{2}^{*}(r)$ and 


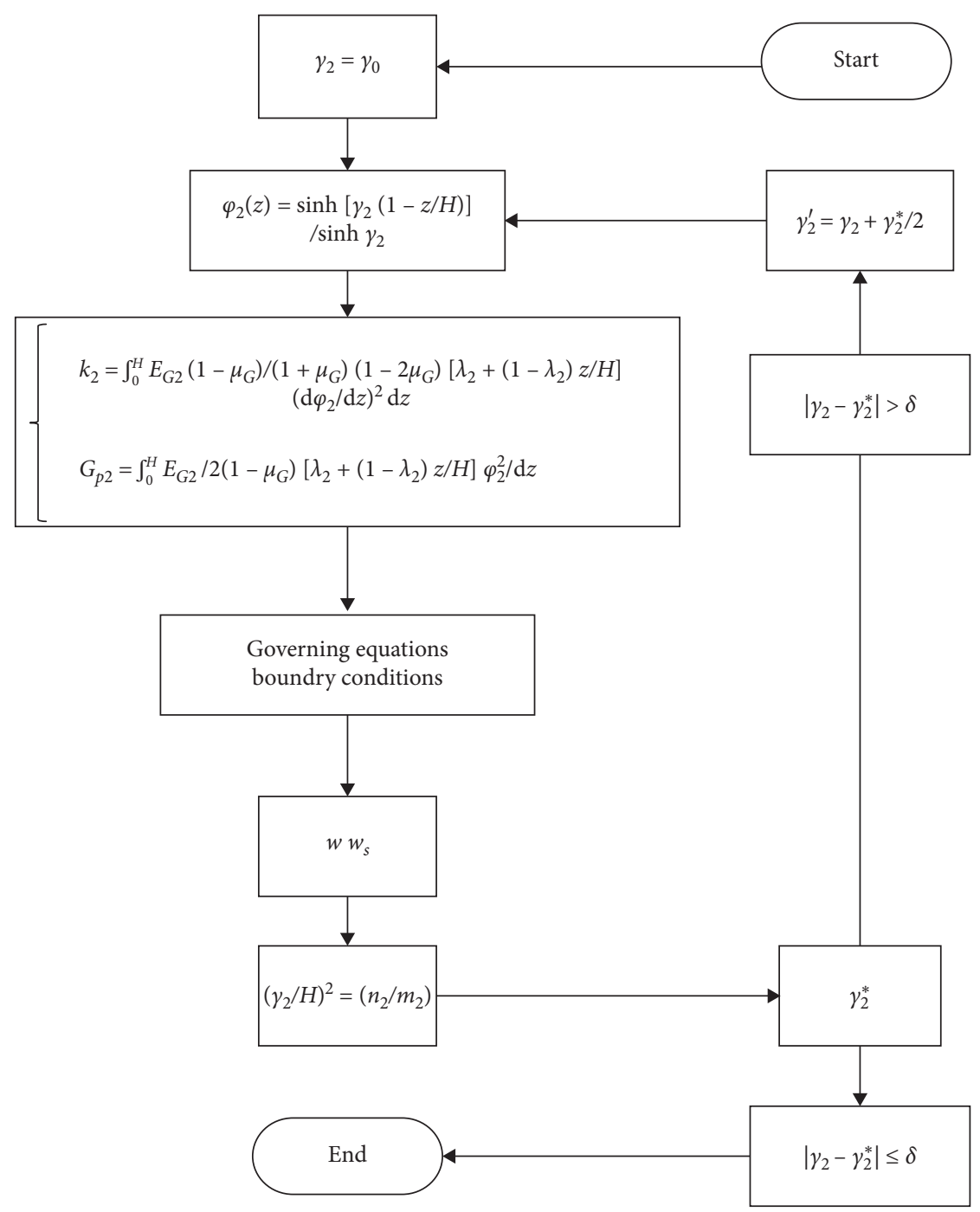

FIGURE 4: Iterative flow chart of the attenuation parameter for the Gibson elastic foundation.

$w_{3}^{*}(r)$ are all zero. Then the complete solutions are expressed as real functions as follows:

$$
\left\{\begin{array}{l}
w_{1}^{*}(r)=\frac{q}{k}+d_{1} s_{0}\left(\frac{r}{l}\right)+d_{2} t_{0}\left(\frac{r}{l}\right)+d_{3} f_{0}\left(\frac{r}{l}\right)+d_{4} g_{0}\left(\frac{r}{l}\right) \\
w_{2}^{*}(r)=d_{5} s_{0}\left(\frac{r}{l}\right)+d_{6} t_{0}\left(\frac{r}{l}\right)+d_{7} f_{0}\left(\frac{r}{l}\right)+d_{8} g_{0}\left(\frac{r}{l}\right) \\
w_{3}^{*}(r)=d_{9} I_{0}(\alpha r)+d_{10} K_{0}(\alpha r)
\end{array}\right.
$$

where $d_{1}, d_{2}, d_{3}, \ldots, d_{9}, d_{10}$ are arbitrary constants. Moreover, $I_{0}$ and $K_{0}$ are modified zero-order Bessel functions of the first and second types, respectively.

4.2. Loads and Boundary Conditions. In this paper, the radius of the uniformly distributed circular load $q$ is classified into two extreme cases and one general case. Thus, the radius of the uniformly distributed load is $b=a, b=0$, and $b \in(0, a)$, respectively. Some equations can be derived using the boundary conditions and continuity conditions for circular thin plates on elastic foundations, and the number of these equations is equal to that of the undetermined coefficients. Accordingly, the simultaneous equations can be solved.

Because both the deflection and rotation angle are finite at the centre of a circle thin plate, it can be concluded that $d_{3}=d_{4}=0$. In the unloaded area of the soil medium, $w_{3}^{*}(+\infty)=0$. Since $I_{0}$ approaches infinity when $r \longrightarrow+\infty$, the constant should be $d_{9}=0$. The remaining seven constants $\left(d_{1}, d_{2}, d_{5}, d_{6}, d_{7}, d_{8}\right.$, and $\left.d_{10}\right)$ are determined using the boundary conditions and the continuous conditions, so that the total solutions for the deflections and internal forces of a circular thin plate on an elastic foundation can be obtained.

\section{Numerical Calculation}

\subsection{Example Analysis}

Example 1. The two modified foundation models are degraded into the traditional Vlasov foundation model, and the characteristic parameters are solved using an iterative procedure. The case of the free circular thin plate on the 
Vlasov elastic foundation subjected to a concentrated load at the plate's centre is analysed. The parameters to be calculated are the following: radius of the circular plate $a=5 \mathrm{~m}$, thickness of the plate $h=0.5 \mathrm{~m}$, elasticity modulus of the plate $E=1.7658 \times 10^{10} \mathrm{~N} / \mathrm{m}^{2}$, Poisson's ratio of the plate $\mu=0.17$, elasticity modulus of the soil $E_{s}=8.6 \times 10^{6} \mathrm{~N} / \mathrm{m}^{2}$, Poisson's ratio of the foundation $\mu_{s}=0.2$, depth of the foundation $H=1 \mathrm{~m}$, and the concentrated load $P=9.81 \times 10^{4} \mathrm{~N}$. The radius of the load is $b=0.01 \mathrm{~m}$, which is adequately small to simulate the concentrated load.

The attenuation parameter $\gamma=0.9836$ can be obtained by programming the mathematical software. On this basis, the foundation reaction coefficient $k^{*}=9.7224 \times 10^{6} \mathrm{~N} / \mathrm{m}^{3}$ and the shear coefficient $G_{p}^{*}=1.0592 \times 10^{6} \mathrm{~N} / \mathrm{m}$ can be further obtained. The deflection value of the circular thin plate at the centre is $0.320 \mathrm{~mm}$, which is consistent with the results of various methods cited in the literature [20]. It proves the reliability of the analysis and calculation in this paper. The deflection of the circular thin plate resting on the traditional Vlasov elastic foundation is shown in Figure 5.

Example 2. On the basis of Example 1, the case of circular thin plates on a transversely isotropic two-parameter foundation subjected to a local distributed load is analysed. The calculated parameters are as follows: radius of circular plate $a=1.35 \mathrm{~m}$, thickness of circular plate $h=0.25 \mathrm{~m}$, elasticity modulus of plate $E=2.45 \times 10^{10} \mathrm{~N} / \mathrm{m}^{2}$, Poisson's ratio of the plate $\mu=1 / 6$, $E_{T 1}=39.2 \mathrm{MPa}, E_{T 2}=29.4 \mathrm{MPa}, \mu_{T 1}=0.4, \mu_{T 2}=0.4, G_{T 2}=$ $14 \mathrm{MPa}$. The depth of the foundation is $H=2 \mathrm{~m}$, and a uniform surface load $q=1 \times 10^{6} \mathrm{~N} / \mathrm{m}^{2}$ with a radius of $b=0.18 \mathrm{~m}$ is applied at the centre area of the circular thin plate.

A very simple computer program has been developed, and the attenuation parameter $\gamma_{1}=1.783$ can be obtained using an iterative technique. On this basis, the foundation reaction coefficient $k_{1}=5.7694 \times 10^{7} \mathrm{~N} / \mathrm{m}^{3}$ and the shear rigidity $G_{p 1}=6.6314 \times 10^{6} \mathrm{~N} / \mathrm{m}$ can be further obtained. The deflection value at the circular plate's centre is $0.333 \mathrm{~mm}$. Figures 6 and 7 depict a deflection diagram and a bending moment diagram of a circular thin plate on transversely isotropic two-parameter foundation, respectively. It is observed in Figure 6 that the deformation of the foundation soils outside the circular thin plate is very small. The foundation soils outside the range of the circular thin plate still have deformations; however, the deformation attenuation speed is very fast.

Example 3. On the basis of Example 1, the scenario in which a uniformly distributed lateral load is applied to the entire circular plate is studied. The parameters of a circular thin plate on the Gibson elastic foundation that are to be calculated are the following: the radius of the circular plate $r=0.5 \mathrm{~m}$, thickness of the plate $h=0.05 \mathrm{~m}$, elasticity modulus of the plate $E=2.8 \times 10^{10} \mathrm{~N} / \mathrm{m}^{2}$, Poisson's ratio of the plate $\mu=0.15$, elasticity modulus at the bottom of the Gibson soil $E_{G 2}=6.9 \times 10^{7} \mathrm{~N} / \mathrm{m}^{2}$, Poisson's ratio of the foundation $\mu_{s}=0.3$, depth of the foundation soil , $H=2 \mathrm{~m}$ and the uniformly distributed load $q=2 \times 10^{6} \mathrm{~N} / \mathrm{m}^{2}$ applied to the whole circular thin plate.

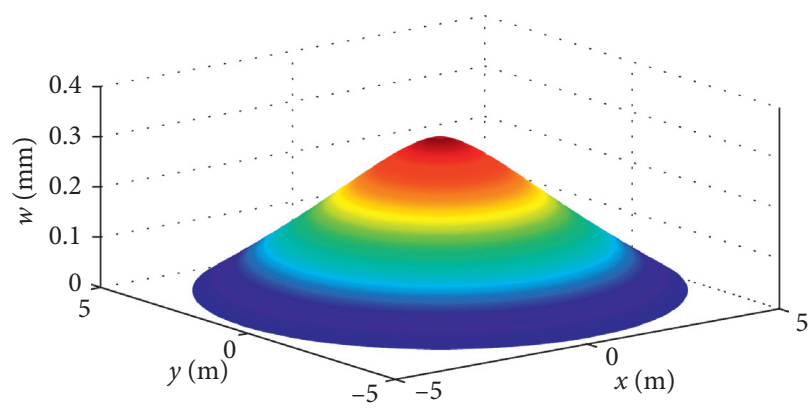

FIgURE 5: Deflection of the circular thin plate on the Vlasov foundation model in Example 1.

Table 1 presents the calculation results of the various parameters and deflections of the circular thin plates, when the characteristic parameters of the Gibson elastic foundation are chosen as $\lambda_{2}=1 / 3$ and $\lambda_{2}=3$, respectively. Further study also shows that the bending deflection of the circular plate on the Gibson elastic foundation is mainly affected by the rigidity of the surface soil; however, it is less affected by the deep part of the foundation.

\subsection{Sensitivity Analysis}

\subsubsection{Transversely Isotropic Two-Parameter Foundation.} Based on Example 2, the effects of soil heterogeneity on the two parameters and the bending of circular thin plates are examined. The variability of the model parameters and deflections, including $k_{1}$ and $w$ for different $E_{T 1}$, is illustrated in Figures 8 and 9. The nonuniformity of the transversely isotropic elastic foundation has a certain influence on various parameters. In a specific range, with the increase in the elasticity modulus $E_{T 1}$ in the transversely isotropic plane, the stiffness coefficient $k_{1}$ of the elastic foundation model increases, and the bending deflection $w$ of the circular thin plate decreases.

5.2.2. Gibson Two-Parameter Foundation. Based on Example 3, the effects of soil heterogeneity on the two parameters and the bending of circular thin plates are studied. The variability of the model parameters and deflections, including $G_{p 2}$ and $w$ for different $\lambda_{2}$, is illustrated in Figures 10 and 11 . The nonuniformity of the Gibson foundation has a certain influence on various parameters. In a specific range, with the increase in the ratio $\lambda_{2}$ of the upper and lower elasticity modulus of the Gibson foundation, shear coefficient $G_{p 2}$ of the elastic foundation model increases and the bending deflection $w$ of the circular thin plate decreases. The research also shows that the harder the surface foundation is, the greater the vertical elasticity coefficient is. This finding demonstrates that the vertical elastic coefficient of the elastic foundation is mainly determined by the rigidity of surface soils. Therefore, to reduce the deflections of elastic foundations in engineering, the physical properties of the Gibson foundation at a certain depth under the foundation can be improved, such as by increasing its strength. Nonetheless, strengthening the deep foundation will have limited results. 


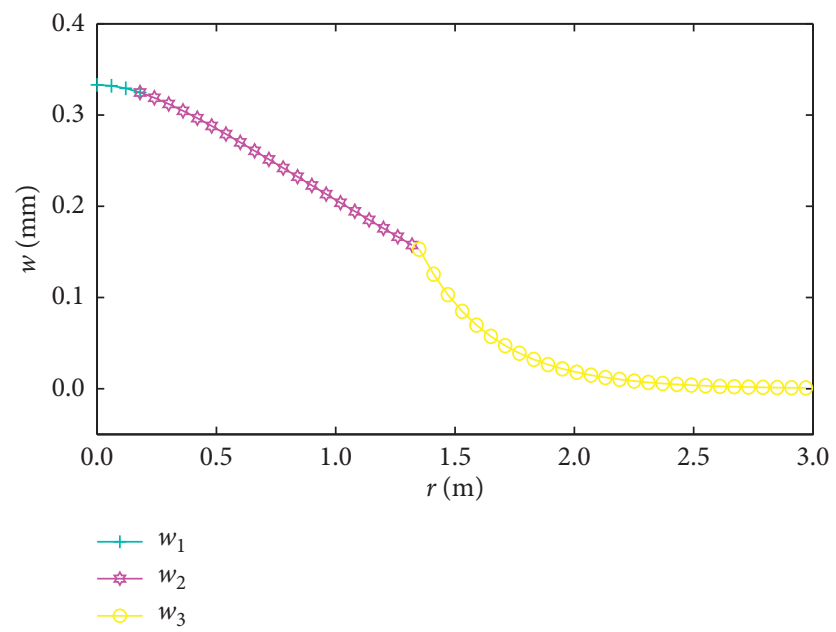

FIGURE 6: Deflection of the circular thin plate and foundation soil in Example 2.

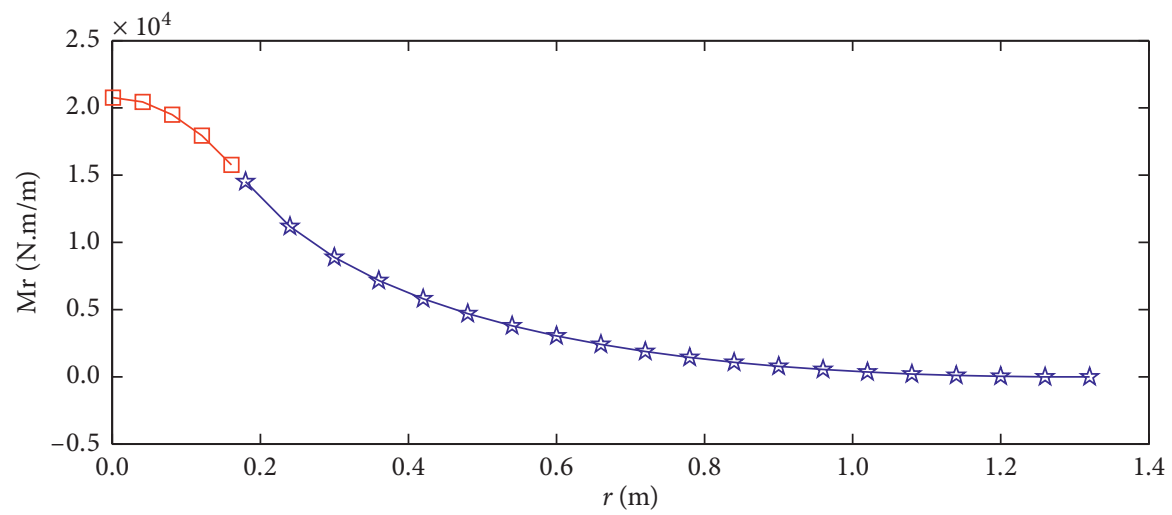

$\square$ Bending moment within the loaded range

B Bending moment outside the loaded range

FIGURE 7: Bending moment of the circular thin plate in Example 2.

TABle 1: Parameters and deflections obtained from the two different foundations in Example 3.

\begin{tabular}{lcccc}
\hline Parameters $\lambda_{2}$ & $\gamma_{2}$ & $k_{2}\left(\mathrm{~N} / \mathrm{m}^{3}\right)$ & $G_{p 2}(\mathrm{~N} / \mathrm{m})$ & $w(\mathrm{~mm})$ \\
\hline $1 / 3$ & 2.1382 & $28,803,000$ & $5,177,600$ & 28.273 \\
3 & 2.1635 & $143,320,000$ & $29,234,000$ & 8.480 \\
\hline
\end{tabular}

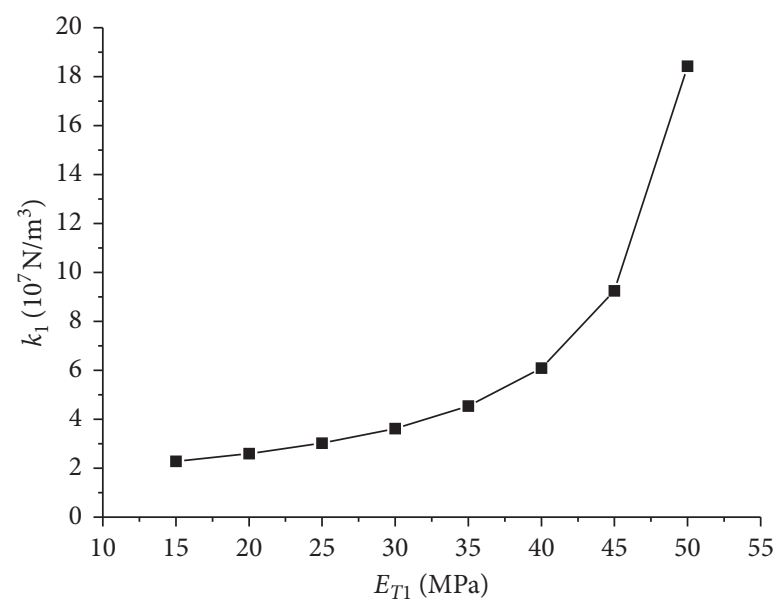

FIgURE 8: Modulus of subgrade reaction $k_{1}$ for different elasticity moduli of the foundation. 


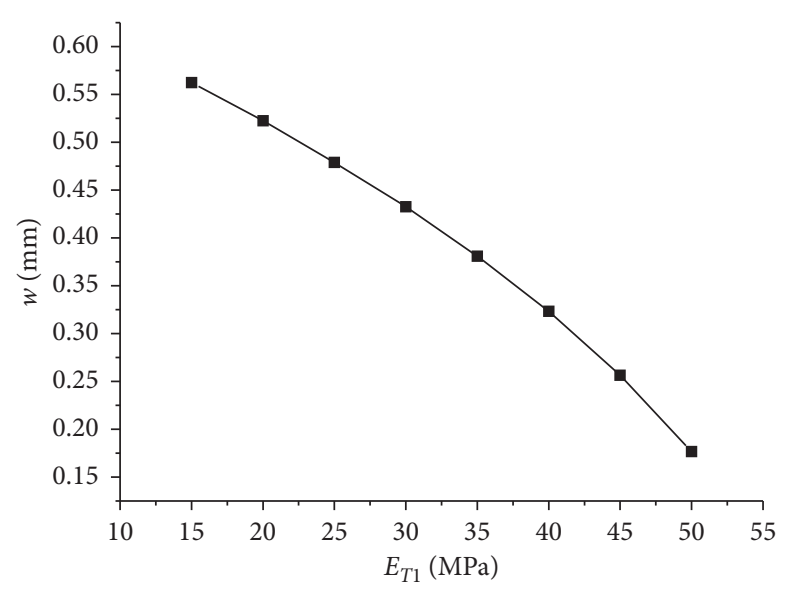

FIgURE 9: Deflection of circular plate $w$ for different elasticity moduli of the foundation.

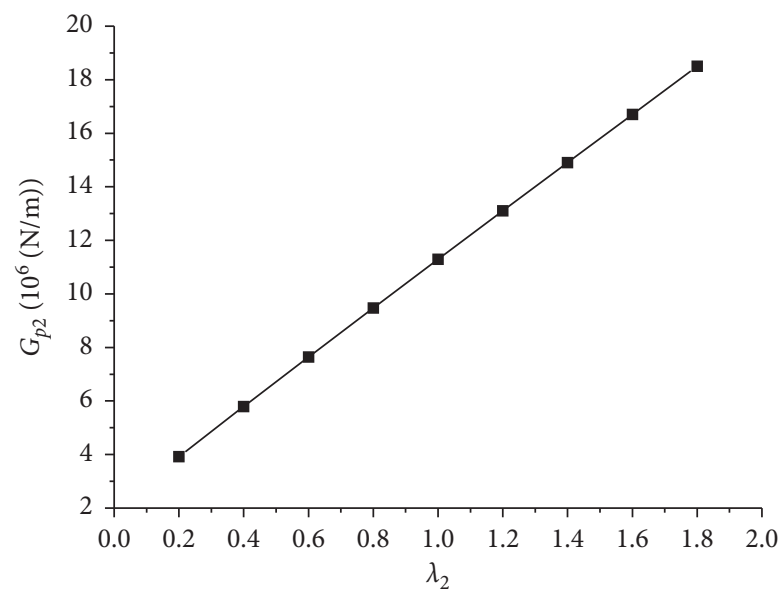

Figure 10: Shear coefficient $G_{p 2}$ for different modulus ratios of the Gibson foundation.

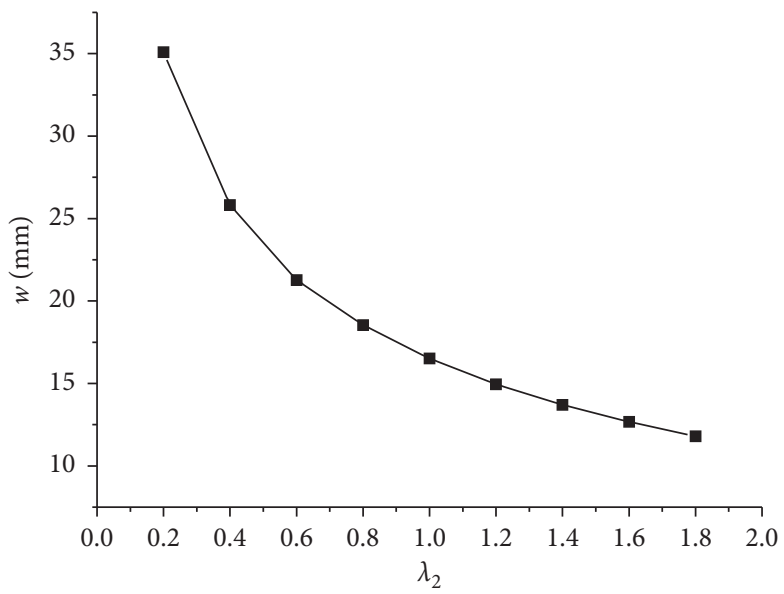

FIgURE 11: Deflection $w$ of the circular plate for different modulus ratios of the Gibson foundation.

5.3. Iterative Analysis. The third parameter $\gamma$ reflects the variation of the soil deformation. To compute the transverse displacement, it is necessary to find $\gamma$, which is not known a priori. The numerical values of the attenuation parameters are shown in Table 2. In the table, $G$ represents the number of iterations of computer programs. $\gamma_{a}$ is the attenuation parameter of a circular thin plate resting on transversely isotropic twoparameter foundations in Example 2. In addition, $\gamma_{b}$ and $\gamma_{c}$ are 
TABLE 2: Attenuation parameters of the two modified Vlasov elastic foundations.

\begin{tabular}{cccccccccc}
\hline$G$ & 0 & 1 & 2 & 3 & $\ldots$ & 9 & 10 & 11 \\
\hline$\gamma_{a}$ & 1.0000 & 1.3200 & 1.5000 & 1.6100 & $\ldots$ & 1.7700 & 1.7830 & - \\
$\gamma_{b}$ & 1.0000 & 1.4273 & 1.6892 & 1.8559 & $\ldots$ & 2.1424 & 2.1508 & 2.1563 & 2.1635 \\
$\gamma_{c}$ & 1.0000 & 1.4816 & 1.7489 & 1.9042 & $\ldots$ & 2.1285 & 2.1382 & - & - \\
\hline
\end{tabular}

the attenuation parameters of a circular thin plate on the Gibson foundations in Example 3 corresponding to $\lambda_{2}=1 / 3$ and $\lambda_{2}=3$, respectively. A start value equal to 1.0 is used here. The input data consist of circular thin plate's properties and the soil properties. The value of $\gamma$ is determined as a function of the characteristics of the plate and the foundation. The program internally calculates the soil parameters using the iterative method. The iterative process is repetitive and the conditions for the end of the cycle must be set. In Figures 3 and 4, the termination criterion is assumed to be $\delta$, a small prescribed value. Eventually the two parameters and transverse displacement will be obtained with the final value of $\gamma$.

\section{Conclusions}

In this study, a sound mathematical model is developed for determining the displacements, bending moments, and shear forces in circular thin plates resting on two modified Vlasov foundations. The influence of heterogeneous soils on the bending of the circular thin plates and the characteristic parameters of two modified Vlasov foundation models is analysed. The presented method can be expanded to consider layered soils, solve the problem of free vibration, and so forth. It is recommended that the similar studies be performed on beams and shells on elastic foundations. The following conclusions are obtained through theoretical derivation and example analyses:

(1) Based on the principle of minimum potential energy, the governing differential equations and boundary conditions for circular thin plates on two modified Vlasov foundations are derived by a variational approach. The equation is determined that the attenuation parameter needs to satisfy using an iterative procedure, which provides a theoretical basis for determining this parameter. The analytical solution for the bending problem of circular thin plates on the two modified Vlasov foundations is established. If the soils of the two modified Vlasov foundations were assumed to be a traditional homogeneous medium, the results in this study would be degraded to the classical case of circular thin plates on traditional two-parameter elastic foundation.

(2) The inhomogeneity of transversely isotropic soils has a certain influence on the deflections, internal forces, and various characteristic parameters of circular thin plates. The results show that the elastic modulus in the transverse isotropic plane, the elastic modulus in the vertical transverse isotropic plane, and the thickness of the soil layer all have a certain influence on the attenuation parameter as well as on the coefficient of subgrade reaction and shear coefficient; however, the influence degree is not the same.

(3) The inhomogeneity of the Gibson soils also has a certain influence on the deflections, internal forces, and various characteristic parameters of circular thin plates. The influence should be considered in practice. The mechanical behaviour of elastic foundations is mainly determined by the characteristics of shallow foundation soils under structures, rather than the deep parts of the foundation soils.

(4) Principles of solid mechanics are used instead of an empirical or experimental evaluation of the attenuation parameter and the coefficient of the subgrade reaction and shear modulus. Only the geometric and material characteristics of heterogeneous soils and structures are used to calculate the attenuation parameters iteratively to obtain two more reliable parameter values. The two modified Vlasov foundation models can describe the continuity of foundation soils. The findings of this research thus enrich and extend the content of the Vlasov foundation model.

\section{Data Availability}

All data used in this study are available upon request from the corresponding author.

\section{Conflicts of Interest}

The authors declare that they have no conflicts of interest.

\section{Acknowledgments}

The authors gratefully acknowledge the support of the National Natural Science Foundation of China (Grant no. 51875463).

\section{References}

[1] Y. Zhang and X. Liu, "Response of an infinite beam resting on the tensionless Winkler foundation subjected to an axial and a transverse concentrated loads," European Journal of Mechanics-A/Solids, vol. 77, Article ID 103819, 2019.

[2] T. Nogami and M. W. O'Neill, "Beam on generalized twoparameter foundation," Journal of Engineering Mechanics, vol. 111, no. 5, pp. 664-679, 1985.

[3] C.-L. Wang, Y. Huang, and J.-H. Jia, "Analytical solutions of steady vibration of a free rectangular plate on the semi-infinite elastic foundation," Chinese Journal of Applied Mathematics and Mechanics, vol. 28, no. 2, pp. 156-164, 2007.

[4] C. V. G. Vallabhan, W. T. Straughan, and Y. C. Das, "Refined model for analysis of plates on elastic foundations," Journal of Engineering Mechanics, vol. 117, no. 12, pp. 2830-2843, 1991. 
[5] C. V. G. Vallabhan and Y. C. Das, "A refined model for beams on elastic foundations," International Journal of Solids and Structures, vol. 27, no. 5, pp. 629-637, 1991.

[6] C. V. G. Vallabhan and Y. C. Das, "Modified Vlasov model for beams on elastic foundations," Journal of Geotechnical Engineering, vol. 29, no. 1, pp. 956-966, 1992.

[7] R. Höller, M. Aminbaghai, L. Eberhardsteiner et al., "Rigorous amendment of Vlasov's theory for thin elastic plates on elastic Winkler foundations, based on the principle of virtual power," European Journal of Mechanics-A/Solids, vol. 73, pp. 449482, 2019.

[8] K. Ozgan, "Dynamic analysis of thick plates including deep beams on elastic foundations using modified Vlasov model," Shock and Vibration, vol. 20, no. 1, pp. 29-41, 2013.

[9] H. Wang, R. Höller, M. Aminbaghai et al., "Concrete pavements subjected to hail showers: a semi-analytical thermoelastic multiscale analysis," Engineering Structures, vol. 200, Article ID 109677, 2019.

[10] Z. Y. Ai and D. L. Feng, "BEM analysis of laterally loaded pile groups in multi-layered transversely isotropic soils," Engineering Analysis with Boundary Elements, vol. 44, pp. 143-151, 2014.

[11] Z. L. Dun and G. B. Liu, "Analytical solution for axial symmetric problems of transversely isotropic foundation under several common loads," Engineering Mechanics, vol. 20, no. 6, pp. 187-194, 2003.

[12] R. Y. Liang and E. S. Shatnawi, "Estimating subgrade reaction modulus for transversely isotropic rock medium," Journal of Geotechnical and Geoenvironmental Engineering, vol. 136, no. 8, pp. 1077-1085, 2010.

[13] R. E. Gibson, "Some results concerning displacements and stresses in a non-homogeneous elastic half-space," Géotechnique, vol. 17, no. 1, pp. 58-67, 1967.

[14] M. Eisenberger and J. Clastornik, "Vibrations and buckling of a beam on a variable winkler elastic foundation," Journal of Sound and Vibration, vol. 115, no. 2, pp. 233-241, 1987.

[15] M. Eisenberger and J. Clastornik, "Beams on variable twoparameter elastic foundation," Journal of Engineering $\mathrm{Me}$ chanics, vol. 113, no. 10, pp. 1454-1466, 1987.

[16] T. Y. Yang, "A finite element analysis of plates on a two parameter foundation model," Computers \& Structures, vol. 2, no. 4, pp. 593-614, 1972.

[17] C. V. G. Vallabhan and Y. C. Das, "Parametric study of beams on elastic foundations," Journal of Engineering Mechanics, vol. 114, no. 12, pp. 2072-2082, 1988.

[18] R. Jones and J. Xenophontos, "The Vlasov foundation model," International Journal of Mechanical Sciences, vol. 19, no. 6, pp. 317-323, 1977.

[19] A. P. S. Selvadurai, Elastic Analysis of Soil-Foundation Interaction, China Railway Publishing House, Beijing, China, 1984.

[20] Y. G. Xiao and D. Feng, "A fast computational algorithm for the element-free Galerkin method for a circular plate on elastic foundation subjected to concentrated load," Chinese Journal of Solid Mechanics, vol. 39, no. 5, pp. 522-529, 2018. 